\title{
Role of protein kinase $C-\delta$ in the regulation of collagen gene expression in scleroderma fibroblasts
}

\author{
Sergio A. Jimenez, ${ }^{1}$ Svetlana Gaidarova, ${ }^{1}$ Biagio Saitta, ${ }^{1}$ Nora Sandorfi, ${ }^{1}$ David J. Herrich, ${ }^{2}$ \\ Joan C. Rosenbloom, ${ }^{2}$ Umberto Kucich, ${ }^{2}$ William R. Abrams, ${ }^{2}$ and Joel Rosenbloom ${ }^{2}$ \\ ${ }^{1}$ Division of Rheumatology, Department of Medicine, Thomas Jefferson University, Philadelphia, Pennsylvania, USA \\ ${ }^{2}$ Department of Anatomy and Histology, University of Pennsylvania, School of Dental Medicine, Philadelphia, Pennsylvania, USA \\ Address correspondence to: Sergio A. Jimenez, Thomas Jefferson University, Division of Rheumatology, \\ 233 S. 10th Street, Room 509 BLSB, Philadelphia, Pennsylvania 19107-5541, USA. \\ Phone: (215) 503-5042; Fax: (215) 923-4649; E-mail: Sergio.Jimenez@mail.tju.edu.
}

Received for publication January 25, 2001, and accepted in revised form September 10, 2001.

\begin{abstract}
Working with cultured dermal fibroblasts derived from control individuals and patients with systemic sclerosis (SSc), we have examined the effects of protein kinase C- $\delta$ (PKC- $\delta$ ) on type I collagen biosynthesis and steady-state levels of COL1A1 and COL3A1 mRNAs. Rottlerin, a specific inhibitor of PKC- $\delta$, exerted a powerful, dose-dependent inhibition of type I and type III collagen gene expression in normal and SSc cells. Optimal rottlerin concentrations caused a 70-90\% inhibition of type I collagen production, $\mathrm{a}>\mathbf{8 0} \%$ reduction in COL1A $1 \mathrm{mRNA}$, and a $>70 \%$ reduction in COL3A1 $\mathrm{mRNA}$ in both cell types. In vitro nuclear transcription assays and transient transfections with COL1A1 promoter deletion constructs demonstrated that rottlerin profoundly reduced COL1A1 transcription and that this effect required a 129 -bp promoter region encompassing nucleotides -804 to -675 . This COL1A1 segment imparted rottlerin sensitivity to a heterologous promoter. Cotransfections of $C O L 1 A 1$ promoter constructs with a dominant-negative PKC- $\delta$ expression plasmid showed that suppression of this kinase silenced COL1A1 promoter activity. The results indicate that PKC- $\delta$ participates in the upregulation of collagen gene transcription in SSc and suggest that treatment with PKC- $\delta$ inhibitors could suppress fibrosis in this disease.
\end{abstract}

J. Clin. Invest. 108:1395-1403 (2001). DOI:10.1172/JCI200112347.

\section{Introduction}

Systemic sclerosis (SSc) is characterized by the excessive deposition of collagen and other connective tissue components in skin and multiple internal organs, prominent and often severe alterations in the microvasculature, and humoral and cellular immunologic abnormalities (for reviews see refs. 1-4). The exact mechanisms involved in the pathogenesis of SSc are not known. However, it is clear that progressive cutaneous, visceral, and vascular fibrosis is responsible for most of its clinical manifestations $(4,5)$. Indeed, SSc severity and mortality are determined by the extent and progression of tissue fibrosis (6).

Despite recent advances in understanding the regulation of collagen gene expression, the mechanisms responsible for the pathologic increase in the expression of collagen genes in SSc have not been elucidated. Fibroblasts from affected SSc skin cultured in vitro produce excessive amounts of various collagens (4, 7, 8 ), and display increased transcription of the corresponding genes $(9,10)$. However, the mechanisms responsible for the increased transcriptional activity of collagen genes in SSc fibroblasts are not fully understood. TGF- $\beta$ plays a crucial role in the development of tissue fibrosis (reviewed in refs. 11,12), and a plausible hypothesis has suggested its involvement in the pathogenesis of SSc (13). The numerous activities of this pleiotropic factor have made it difficult to elucidate the exact mechanisms by which it exerts its fibrogenic effects, although it has been demonstrated that the intracellular pathways involved are mediated through complex interactions between specific cell-surface receptors and several intracellular proteins of the Smad family (reviewed in refs. 14, 15).

We recently examined other intracellular signaling constituents through which TGF- $\beta$ may upregulate the expression of matrix protein genes, and identified phosphatidylcholine-specific phospholipase $\mathrm{C}$, protein kinase $\mathrm{C}-\delta(\mathrm{PKC}-\delta)$, geranylgeranyl transferase $\mathrm{I}$, and $\mathrm{p} 38$ as possible participants (16-18). These findings suggested that one or more of these proteins could also be involved in the upregulation of collagen gene expression in SSc. Indeed, we recently demonstrated that inhibition of geranylgeranyl transferase I caused a profound and selective inhibition of expression of type I and type III collagen genes in normal and SSc dermal fibroblasts (19).

Here, we examined the effects of rottlerin, a specific inhibitor of PKC- $\delta(20,21)$, on the expression of type I and type III collagen genes (COL1A1 and COL3A1, respectively) in normal and SSc dermal fibroblasts in vitro. Rottlerin inhibited COL1A1 and COL1A3 expression in a dose-dependent manner in both cell types, without affecting the expression of other genes examined. The inhibitory effects of rottlerin on COL1A1 
expression resulted from a profound reduction in COL1A1 transcription that was exerted through a 129bp region of the gene promoter. Studies to examine the possibility that PKC- $\delta$ is involved in the pathogenesis of the upregulated collagen gene expression in SSc showed that SSc cells displayed higher levels of immunoreactive protein when examined by immunofluorescence and Western analysis. Furthermore, cotransfection of SSc cells with COL1A1 promoter constructs and a dominant-negative PKC- $\delta$ expression construct showed a reduction of COL1A1 promoter activity. These results indicate that PKC- $\delta$ may be involved in the upregulated expression of interstitial collagen genes in SSc, and suggest that specific inhibitors of this enzyme should be further investigated as potential therapeutic approaches for this devastating and incurable disease.

\section{Methods}

Fibroblast cultures. Dermal fibroblast cell lines were established from patients with SSc who fulfilled the American College of Rheumatology criteria for the classification of SSc (22). To avoid variability introduced by heterogeneity in the clinical characteristics and the stage of evolution of SSc, only cells from untreated patients with diffuse SSc of recent onset and rapid progression were studied, as described previously $(8,10,19)$. The cell lines were obtained from full-thickness skin biopsies surgically excised for diagnostic purposes from the leading edge of clinically apparent SSc lesions. Normal fibroblasts were obtained from age- and sex-matched individuals undergoing surgical procedures. All studies were approved by the Institutional Review Board of Thomas Jefferson University. Fibroblast cultures were established as described previously $(8,10,19)$, and were cultured in DMEM supplemented with 10\% FBS in 5\% $\mathrm{CO}_{2}$ for 4 days until they reached confluence.

For all studies, only early passage $(<12)$ fibroblasts were used to avoid changes in their original phenotype during subculture. Cells were maintained in DMEM containing 10\% FBS (Life Technologies Inc., Grand Island, New York, USA), $1 \%$ vitamins, $2 \mathrm{mM}$ glutamine, antibiotics, and Fungizone, and were incubated at $37^{\circ} \mathrm{C}$ in a $5 \% \mathrm{CO}_{2}$ humidified atmosphere. The cultures were supplemented with $50 \mu \mathrm{g} / \mathrm{ml}$ ascorbic acid for 24 hours to optimize their level of collagen production. At the conclusion of all experiments, the cells were counted and their viability was assessed using trypan blue exclusion. High-resolution fluorescence immunomicroscopy. Fibroblasts $(40,000)$ were plated on coverslips and maintained in 10\% FBS in DMEM for 24 hours. When the cells reached confluence, they were prepared for analysis with high-resolution fluorescence immunomicroscopy. Cells were washed in PBS and fixed in $2 \mathrm{ml}$ of freshly prepared 3\% paraformaldehyde for 10 minutes. After the fixative was washed off with PBS, the cells were permeabilized with $0.05 \%$ Triton X-100 in PBS. Nonspecific binding was blocked with $4 \%$ normal goat serum in PBS with $0.5 \%$ Triton $X-100$. The cells were incubated for 30 minutes with anti-PKC- $\delta$ antibodies at a 1:10 dilution. After washing off the primary antibody, a 1:100 dilution of fluorescein-conjugated goat anti-rabbit IgG (Vector Laboratories Inc., Burlingame, California, USA) was added to the coverslips; after 30 minutes these were mounted on slides using the SlowFade Light Antifade Kit from Molecular Probes Inc. (Eugene, Oregon, USA). All staining steps were carried out at $37^{\circ} \mathrm{C}$. Fluorescence was visualized using excitation filters at 450-490 $\mathrm{nM}$ and emission filters at 500-550 $\mathrm{nM}$ with a 495 LP dichroic filter (Chroma Technology Corp., Brattleboro, Vermont, USA). IPLab scientific imaging software (Scanalytics Inc., Fairfax, Virginia, USA) was used for image and acquisition analysis.

Western analysis of PKC- $\delta$ in cell lysates. To examine the amounts of immunoreactive PKC- $\delta$ in cell lysates, $100-\mathrm{ml}$ dishes of confluent normal and SSc cells were washed three times with $25 \mathrm{ml}$ of cold PBS; subsequently the monolayers were lysed in $250 \mu \mathrm{l}$ of lysis buffer $(10 \mathrm{mM}$ Tris-HCl at $\mathrm{pH} 7.25,1 \mathrm{mM} \mathrm{MgCl}_{2}, 0.2 \%$ Triton X-100) containing protease inhibitors and $1 \mathrm{mM}$ PMSF. The suspension was sonicated and then centrifuged at $11,000 \mathrm{~g}$ for 10 minutes at $4^{\circ} \mathrm{C}$. Aliquots containing $80 \mu \mathrm{g}$ of lysate were boiled for 10 minutes and electrophoresed on $7.5 \%$ polyacrylamide gels (Bio-Rad Laboratories Inc., Hercules, California, USA) at $200 \mathrm{~V}$ for 1 hour. Following electrophoresis, the blots were transferred to an ULTRAN membrane (Schleicher and Schuell, Dassel, Germany), blocked for 2 hours with $5 \%$ powdered milk dissolved in PBS and $0.1 \%$ Tween 20 , and then incubated overnight at $4^{\circ} \mathrm{C}$ with $4 \mu \mathrm{g} / \mathrm{ml}$ of a rabbit polyclonal IgG anti-PKC- $\delta$ antibody (Upstate Biotechnology Inc., Lake Placid, New York, USA). The immunoreactive proteins were visualized using a goat anti-rabbit secondary antibody conjugated to horseradish peroxidase using the ECL chemiluminescence detection system (Amersham Pharmacia Biotech, Buckinghamshire, England).

Treatment of cultured fibroblasts with rottlerin. Rottlerin was purchased from Biomol Research Laboratories Inc. (Plymouth Meeting, Pennsylvania, USA). All other chemicals were of reagent grade. Four strains of SSc fibroblasts and two strains of normal fibroblasts were studied. The cells were plated in 60-mm Falcon plastic culture dishes (Becton Dickinson and Co., Lincoln Park, New Jersey, USA) at a density of 150,000 cells per dish, and cultured until confluent. For dose-response studies, the cells were incubated with $0.5-3.0 \mu \mathrm{M}$ rottlerin in $1 \%$ FBS for 24 hours. All subsequent experiments were performed with $3 \mu \mathrm{M}$ rottlerin, a dose found to have the maximum inhibitory effect on collagen gene expression.

Fibroblast collagen biosynthesis. Confluent fibroblast cultures were used to examine the effects of rottlerin on optimal fibroblast collagen biosynthesis as described previously $(23,24)$. The medium was removed and 1.5 $\mathrm{ml}$ of fresh DMEM containing $3 \mu \mathrm{M}$ rottlerin, $10 \% \mathrm{FBS}$, $50 \mu \mathrm{g} / \mathrm{ml}$ ascorbic acid, $100 \mu \mathrm{g} / \mathrm{ml} \beta$-aminoproprionitrile, and $5 \mu \mathrm{Ci} / \mathrm{ml}\left[{ }^{14} \mathrm{C}\right]$ proline (specific activity: 247 $\mathrm{mCi} / \mathrm{mM}$; NEN Life Science Products, Boston, Massachusetts, USA) was added. At various intervals after incubation with $\left[{ }^{14} \mathrm{C}\right]$ proline, the medium was removed 
and the following reagents were added, to the indicated final concentrations: $0.15 \mathrm{M} \mathrm{NaCl}, 0.05 \mathrm{M}$ Tris- $\mathrm{HCl}, 4$ $\mathrm{mM}$ EDTA, and $10 \mu \mathrm{g} / \mathrm{ml}$ PMSF. The samples were then dialyzed against $0.15 \mathrm{M} \mathrm{NaCl}, 0.05 \mathrm{M}$ Tris- $\mathrm{HCl}, \mathrm{pH}$ 7.4 buffer containing the same concentration of protease inhibitors. The monolayers containing cells and adherent protein were solubilized in $1 \mathrm{M} \mathrm{NaCl}, 0.05 \mathrm{M}$ Tris-HCl, pH 7.4 buffer containing $4 \mathrm{mM}$ EDTA and 10 $\mu \mathrm{g} / \mathrm{ml}$ PMSF, and the solutions were dialyzed as described for the media. The amount of radiolabeled (newly synthesized) collagen was measured by a collagenase digestion assay (25) using pure bacterial collagenase (BRL Laboratories Inc., Bethesda, Maryland, USA) in the presence of protease inhibitors. In certain experiments, both media and cell layers were analyzed. However, in most experiments, only the culture media were studied, because as shown previously, more than $85 \%$ of the total collagen synthesized by both the control and the experimental cultures was released into the media under the conditions used $(23,24)$.

Determination of type I collagen production. The type I collagen present in culture media was quantified by an indirect ELISA (26) using a human collagen type I polyclonal antibody (BIODESIGN International, Kennebunk, Maine, USA). A standard curve was established relating the inhibition of color development to the concentration of added antigen (human type I collagen; Southern Biotechnology Associates, Birmingham, Alabama, USA). The concentration of type I collagen in the cell culture supernatants was analyzed in duplicate and was determined directly from the standard inhibition curve, which was linear between 4 and $250 \mathrm{ng} / \mathrm{ml}$ of type I collagen. The interassay variation was $10-15 \%$, and the intra-assay variation was $5-10 \%$.

Northern bybridizations. Fibroblasts were grown to confluence. Following incubation either under control conditions or with rottlerin, total RNA was isolated by acid guanidinium thiocyanate-phenol-chloroform extraction (27). Aliquots of total RNA (5-10 $\mu \mathrm{g} /$ well) were electrophoresed on formaldehyde $1.2 \%$ agarose gels. The RNA was then transferred to Zeta-Probe membranes (Bio-Rad Laboratories Inc., Richmond, Virginia, USA) by vacuum blotting, and the filters were hybridized to ${ }^{32} \mathrm{P}$-radiolabeled human cDNA for COL1A1, COL3A1, and cyclooxygenase-1 as described previously (19). Equivalent amounts of RNA were loaded, and RNA loading and transfer were evaluated by probing with a GAPDH cDNA. Equivalent loading and transfer were also verified by quantitative image analysis of ethidium bromide staining of ribosomal RNA in the same blots. The filters were analyzed using phosphor imaging eqiuipment (ImageQuant version 5.1 software, Molecular Dynamics, Sunnyvale, California, USA). All mRNA analyses were performed in duplicate.

In vitro nuclear transcription assay. The transcription rates of COL1A 1 and COL1A3 were measured by an in vitro nuclear run-off assay. Fibroblasts were cultured in T-175 flasks for 24 hours in the presence or absence of $3 \mu \mathrm{M}$ rottlerin. At the end of the incubation period, cell layers were trypsinized, nuclei were isolated, and transcription reactions were carried out exactly as described previously (28), except that after hybridizations the filters were analyzed using storage phosphor technology (ImageQuant version 5.1 software).

Transient transfections of normal and SSc fibroblasts. Transient transfections and cotransfections of normal and SSc fibroblasts were performed using the lipid-based Fu Gene 6 Kit (Roche Diagnostic Corp., Indianapolis, Indiana, USA) as described previously (29). Normal and SSc fibroblasts were plated at $70 \%$ confluence in $60-\mathrm{mm}$ dishes. The medium was changed the next day, and 3 hours later the cells were transfected with a total of 2.5 $\mu \mathrm{g}$ of each plasmid. COL1A1 transcription was assessed using several deletion constructs of the COL1A1 promoter fused to the chloramphenicol acetyl transferase reporter gene (CAT). All the constructs used end at nucleotide +42 to assure transcription in a proper reading frame; their $5^{\prime}$ ends are at $-804,-675,-174$, and -84 bp. The detailed procedures for preparation of these constructs have been described previously $(30,31)$.

Cotransfections with constructs expressing wild-type PKC- $\delta$ or a dominant-negative form of the enzyme (32) were performed using $0.4 \mu \mathrm{g}$ of either bp -804 or bp -675 COL1A1-CAT constructs, and $0.4 \mu \mathrm{g}$ of either of the cotransfected PKC- $\delta$ constructs. Following transfections, fresh medium with or without rottlerin was added; the cells were harvested 48 hours after transfection and lysed by sonication. Total protein content of the cytoplasmic extracts was measured by the Bradford procedure (33). CAT activity in cell extracts was determined by TLC (34). The efficiency of transfection was normalized by cotransfecting $2 \mu \mathrm{g}$ of vector containing $E$. coli $\beta$-galactosidase cDNA (pCMVß-galactosidase; CLONTECH Laboratories, Palo Alto, California, USA) followed by assays of $\beta$-galactosidase enzymatic activity.

Cloning of the rottlerin-responsive element (RRE) upstream of a thymidine kinase heterologous promoter. The construct SJRott804 was generated by PCR amplification of the region of the human COL1A1 promoter encompassing nucleotides -675 to -804 . This region was cloned into pCRII (Invitrogen Corp., San Diego, California, USA), and $\mathrm{XbaI}$ fragments were cloned immediately upstream of the herpes simplex virus thymidine kinase (TK) promoter of pBLCAT2 (CAT2), as described previously (35). The plasmid SJRott804 was sequenced to confirm its sequence and orientation relative to the TK promoter. The construct was transfected into one line of SSc cells, and following transfection the cells were treated with $3 \mu \mathrm{M}$ rottlerin as described above. CAT activity was determined as described above.

\section{Results}

Effects of rottlerin on collagen production and biosynthesis in normal and SSc dermal fibroblasts. To determine the effect of inhibition of PKC- $\delta$ on the production of type I collagen by normal and SSc fibroblasts, replicate confluent cultures of normal and SSc fibroblasts were incubated with $0.5-3.0 \mu \mathrm{M}$ rottlerin, after which the col- 

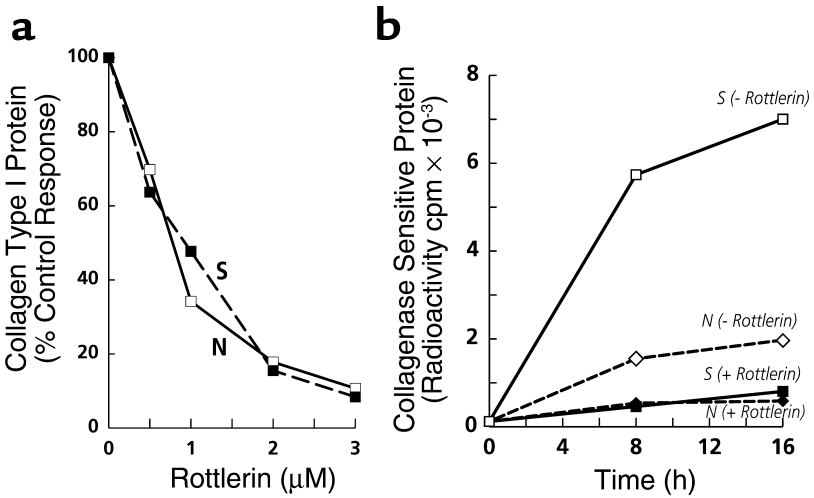

Figure 1

Effect of inhibition of PKC- $\delta$ by rottlerin on type I collagen production and biosynthesis. (a) Confluent cultures of normal (open squares) and SSc (filled squares) dermal fibroblasts were incubated for 24 hours with the indicated concentrations of rottlerin, as described in Methods. The culture media were analyzed for type collagen I using specific ELISA. Values represent the average of duplicate cultures determined in duplicate. (b) Confluent cultures of normal (diamonds) and SSc (squares) dermal fibroblasts were incubated under control conditions (open symbols) or with $3 \mu \mathrm{M}$ rottlerin (filled symbols) and labeled with $\left[{ }^{14} \mathrm{C}\right]$ proline, as described in Methods. Two plates from each set were harvested 8 hours and 16 hours after labeling, and the amount of radiolabeled collagen representing newly synthesized protein present in the media was determined by a collagenase digestion assay.

lagen secreted into the culture media was analyzed by a specific ELISA. Rottlerin decreased the production of type I collagen by both normal and SSc fibroblasts in a concentration-dependent manner, so that at $3 \mu \mathrm{M}$ there was approximately $90 \%$ inhibition (Figure 1a). Biosynthetic studies demonstrated that rottlerin caused a profound reduction in the amounts of newly synthesized collagen (Figure 1b). The amount of radiolabeled collagenase-sensitive protein, representing newly synthesized collagen, was nearly fourfold greater in SSc cells than in normal cells $(7,005 \mathrm{cpm}$ vs. $1,974 \mathrm{cpm}$, respectively). Rottlerin at the optimal concentration $(3 \mu \mathrm{M})$ reduced newly synthesized collagen by about $90 \%$ in the SSc cells, and by about $70 \%$ in normal cells.

Effects of rottlerin on type I and type III collagen gene expression in normal and SSc cells. Total RNA from normal and SSc cells cultured under control conditions or in the presence of increasing concentrations of rottlerin was analyzed by Northern hybridizations for COL1A1 transcripts. SSc cells contained greater levels of COL1A1 mRNA than normal cells; rottlerin caused a concentration-dependent reduction in these levels, with a reduction of approximately $70-90 \%$ at a $3-\mu \mathrm{M}$ concentration in both normal and SSc fibroblasts (Figure 2).

To examine the consistency of rottlerin effects, several normal and SSc fibroblast cell line cultures were incubated with $3 \mu \mathrm{M}$ rottlerin, and COL1A1 expression was examined. Rottlerin uniformly caused a marked decrease of COL1A1 expression in the two normal and four SSc cell lines tested. At the protein level, collagen production was inhibited by $90 \%$ in the normal cells, and by $73-86 \%$ in the SSc cells (Figure 3a). Northern hybridizations confirmed the inhibitory effect; the steady-state $\alpha 1$ (I) collagen mRNA levels were reduced by $88 \%$ and $98 \%$ in the two normal cell lines, and by 83-99\% in the four SSc cell lines (Figure 3b). The effects of $3 \mu \mathrm{M}$ rottlerin on mRNA levels of COL3A1 were also examined in the four SSc cell lines, and inhibition ranging from $66 \%$ to $85 \%$ was found (Figure 4 ).

To determine whether the effects of rottlerin on the expression of collagen genes were selective, mRNA levels for cyclooxygenase-1, a constitutively expressed gene, were also examined. Rottlerin did not cause appreciable changes in cyclooxygenase- 1 transcripts in any of the normal (not shown) or SSc cells (Figure 5) examined. Furthermore, the levels of GAPDH were not affected by rottlerin in either normal or SSc cells (Figures 2-5).

Effect of rottlerin on COL1A1 transcription. SSc fibroblasts obtained from two different individuals (S1 and S2) were treated with $3 \mu \mathrm{M}$ rottlerin. Subsequently, their nuclei were isolated and used for assessment of the rates of transcription of types I and III collagen genes. The results (Figure 6) showed that the transcription of COL1A1 was decreased by $70 \%$ in cell line $\mathrm{S} 1$, and by $53 \%$ in cell line S2. Transcription of COL3A1 was inhibited by more than $95 \%$ in both cell lines. In contrast, the transcription of the GAPDH gene was only minimally affected.

Identification of the COL1A1 promoter region responsive to rottlerin. To identify the region of the COL1A1 promot-
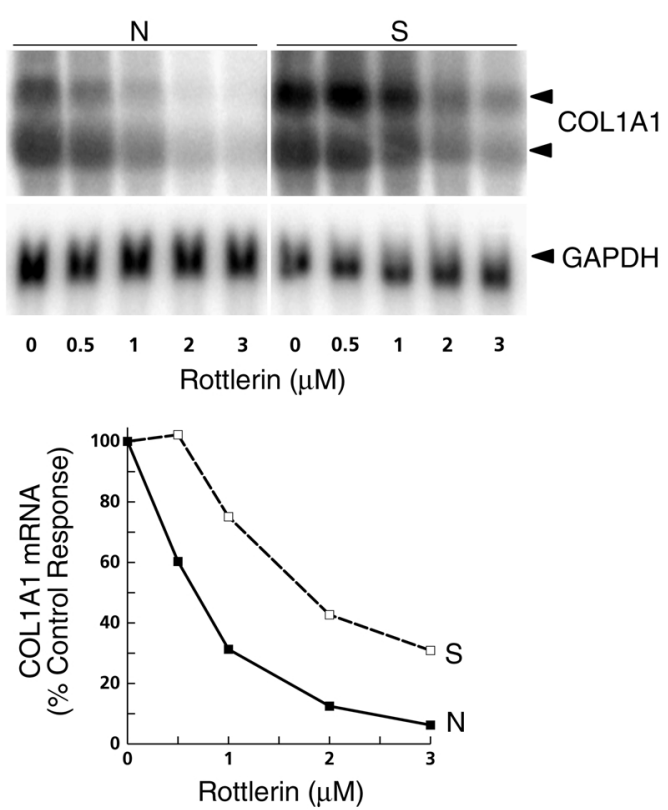

\section{Figure 2}

Effects of inhibition of PKC- $\delta$ on type I collagen mRNA steady-state levels. Total RNA prepared from normal (filled squares) or SSc (open squares) cells treated with various concentrations of rottlerin for 24 hours was subjected to Northern hybridization analysis using COL1A1 cDNA as described in Methods. The two COL1A1 transcripts are indicated by arrowheads. The Northern blots were analyzed by phosphor storage technology; the values shown represent percentage of control (untreated) values. 


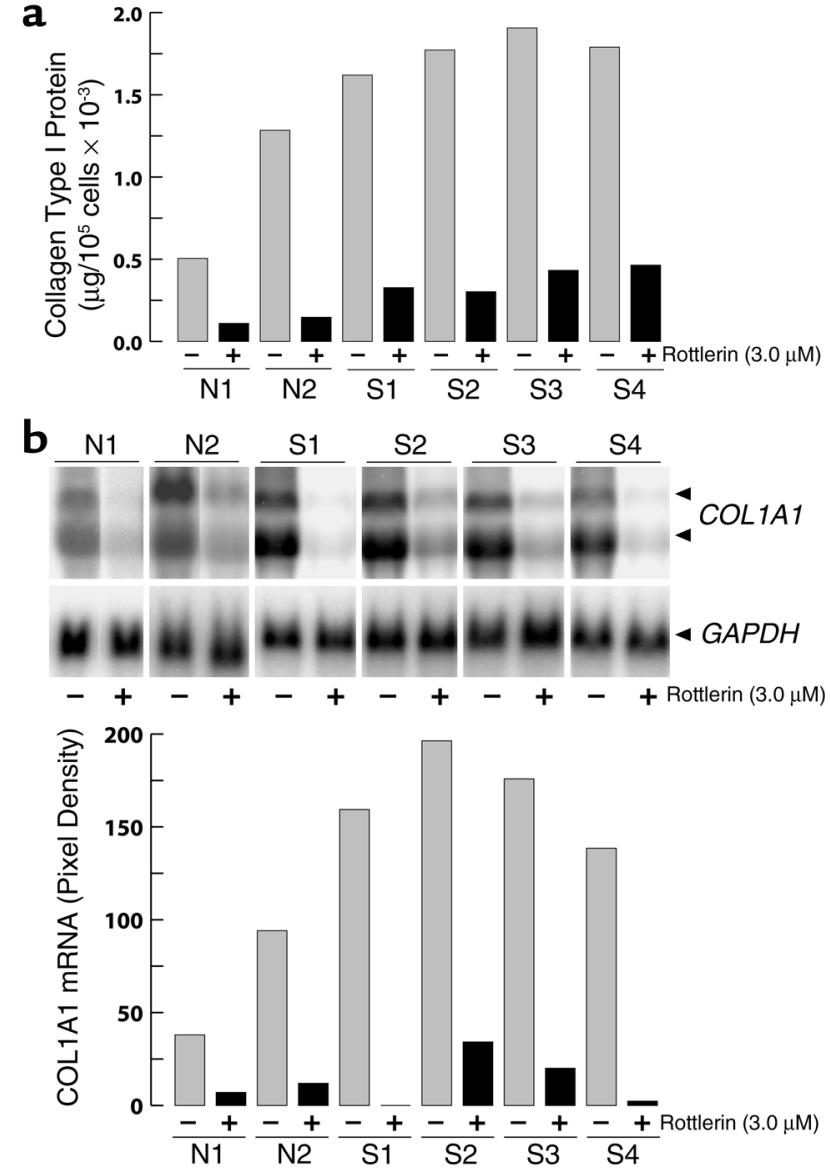

Figure 3

Effects of rottlerin on type I collagen production and COL1A1 steadystate mRNA levels by several cell lines of normal and SSc dermal fibroblasts. (a) Confluent cultures of normal (N) and SSc (S) dermal fibroblasts were incubated for 24 hours with or without $3 \mu \mathrm{M}$ rottlerin, and type I collagen secreted into the media was determined by specific ELISA. Values represent the average of duplicate cultures determined in duplicate. (b) Confluent cultures of normal (N) and SSc (S) dermal fibroblasts were incubated for 24 hours with or without $3 \mu \mathrm{M}$ rottlerin, and the levels of COL1A1 mRNA were determined by Northern hybridization analysis. The two COL1A1 transcripts are indicated by arrowheads. Phosphor image analysis of the Northern blots is shown in actual pixel density after correction for the values obtained with GAPDH hybridizations. Values represent the average of duplicate cultures.

er that is responsive to rottlerin effects, untreated and rottlerin-treated SSc fibroblasts were transiently transfected with gene constructs containing various deletions of the COL1A1 promoter ligated to the CAT reporter gene. The results (Figure 7a) demonstrated that the promoter activity of $\mathrm{bp}-174$ or $\mathrm{bp}-675$ constructs was not affected, whereas that of the bp -804 construct was decreased by more than $85 \%$ following treatment with rottlerin. These results indicated that the inhibitory effect of rottlerin on COL1A1 promoter transcriptional activity was exerted through the 129-bp promoter region encompassing nucleotides -675 to -804 .

To confirm that the COL1A1 promoter region from bp -675 to -804 was necessary and sufficient for rott- lerin-inducible effects on transcription of the gene, the fragment of the COL1A1 promoter encompassing the putative rottlerin-responsive region was subcloned upstream of the herpes simplex virus TK promoter-CAT transcription unit, and was transfected into SSc cells. The results (Figure $7 \mathrm{~b}$ ) demonstrated that rottlerin caused a $75 \%$ reduction in CAT activity, driven by the heterologous TK promoter when the COL1A1 promoter segment encompassing nucleotides -675 to -804 was cloned upstream of the TK promoter.

Inbibition of COL1A1 transcription by dominant-negative $P K C-\delta$ expression. To provide independent evidence for the role of PKC- $\delta$ in the regulation of collagen gene expression, normal and SSc cells were cotransfected with COL1A1 promoter constructs and with expression constructs coding for wild-type or dominant-negative PKC- $\delta$ (32). The results demonstrated that compared with the wild-type PKC- $\delta$, the dominant-negative PKC- $\delta$ inhibited the promoter activity of the bp -804 construct in both the normal (40\% inhibition) and SSc cells (54\% inhibition) (Figure 8). Significantly, the promoter activity of the bp -675 construct was unaffected by expression of dominant-negative PKC- $\delta$.

$P K C-\delta$ expression in cultured normal and SSc fibroblasts. The above findings suggested that increased expression of PKC- $\delta$ may play an important role in stimulating collagen production in SSc cells. Therefore, the levels of PKC- $\delta$ in normal and SSc cells were compared by high-resolution immunomicroscopy and Western
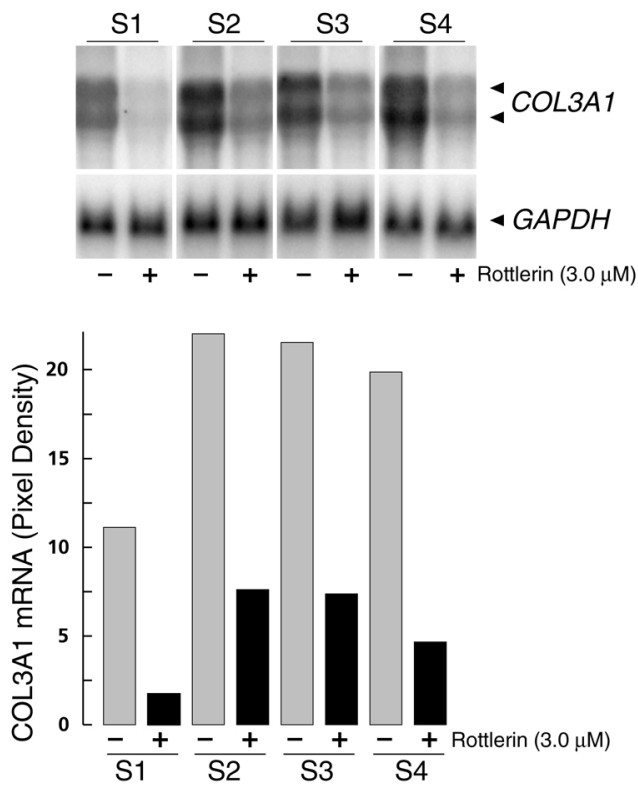

Figure 4

Effect of rottlerin on COL3A1 mRNA levels in SSc dermal fibroblasts. Confluent cultures of SSc fibroblasts (S1-S4) were incubated for 24 hours with or without $3 \mu \mathrm{M}$ rottlerin, and the levels of COL3A1 mRNA were determined by Northern hybridization analysis. The two COL3A1 transcripts are indicated by arrowheads. Densitometric analysis of the Northern blots is shown in actual pixel density after correction for the values obtained with GAPDH hybridizations. Values represent the average of duplicate cultures. 

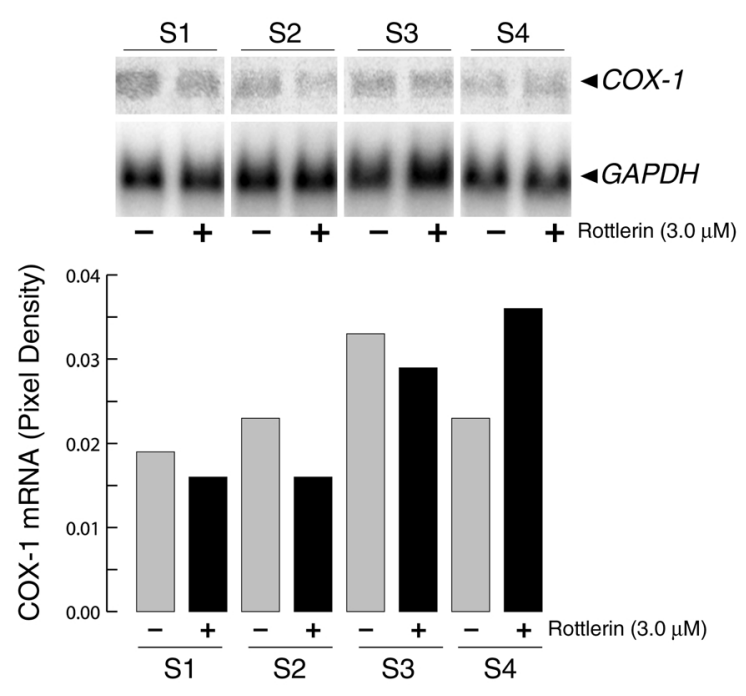

Figure 5

Effect of rottlerin on cyclooxygenase- 1 expression. Confluent cultures of SSc dermal fibroblasts (S1-S4) were incubated for 24 hours with or without $3 \mu \mathrm{M}$ rottlerin, and cyclooxygenase- 1 and GAPDH levels were determined by Northern hybridizations as described in Methods. Phosphor image analysis of the Northern blots is shown in actual pixel density after correction for the values obtained with GAPDH hybridization. Values represent the average of duplicate cultures. COX-1, cyclooxygenase- 1 .

analysis. The apparent level of expression of PKC- $\delta$ epitopes was greater in SSc cells than in normal cells, as observed by high-resolution immunofluorescence microscopy (Figure 9). Western analyses also showed significantly higher PKC- $\delta$ immunoreactivity in cell lysates from three SSc cell isolates than in those from three normal controls (Figure 10).

\section{Discussion}

Tissue fibrosis is the hallmark of numerous human diseases such as scleroderma, pulmonary fibrosis, liver cirrhosis, and graft-versus-host disease, among others. The most prominent biochemical manifestation of these fibrotic processes, also known as fibroproliferative diseases, is an abnormal and exaggerated accumulation of extracellular matrix macromolecules, in particular of type I and type III interstitial collagens.

The mechanisms involved in the regulation of expression of the genes encoding the interstitial collagens under physiologic conditions or during normal tissue repair have not been completely elucidated. Even more elusive have been the pathogenetic steps that lead to increased expression of these genes in abnormal fibrogenesis, such as that occurring in the fibroproliferative diseases. Numerous studies have implicated members of the TGF- $\beta$ family of proteins as playing a crucial role in the initiation and progression of pathologic fibrogenesis (reviewed in refs. 11, $12)$. The TGF- $\beta$ proteins are pleiotropic in their biological activities, modulating cell growth and differentiation as well as extracellular matrix deposition and degradation. This wide range of activities is initiated following interactions of the active TGF- $\beta$ proteins with specific transmembrane receptors, which posses both constitutive (type II) and activated (type I) serinethreonine kinase activity (reviewed in refs. 14, 15, 36).

Although the varied biological activities of the TGF- $\beta$ s have been well documented, the mechanisms through which these molecules modulate the expression of the numerous target genes have been only partially elucidated. Of particular importance are the intracellular transduction pathways that transmit cellsurface signals to the transcriptional units of specific genes, and how these signals provide the selectivity required to influence only certain genes under given conditions. Since the TGF- $\beta$ family is involved in the regulation of a wide variety of fundamental cell processes, it is to be expected that the signaling mechanisms by which these effects are achieved are complex. The discovery that Smad proteins are key intermediates in the signaling process linking TGF- $\beta$ receptors to cellular responses has revealed important new information on the way cells respond to this cytokine (reviewed in refs. 14, 15). These studies have established a basic framework upon which a comprehensive understanding of the molecular events required for TGF- $\beta$-induced modulation of gene expression can be constructed. However, current understanding of these pathways is far from complete.
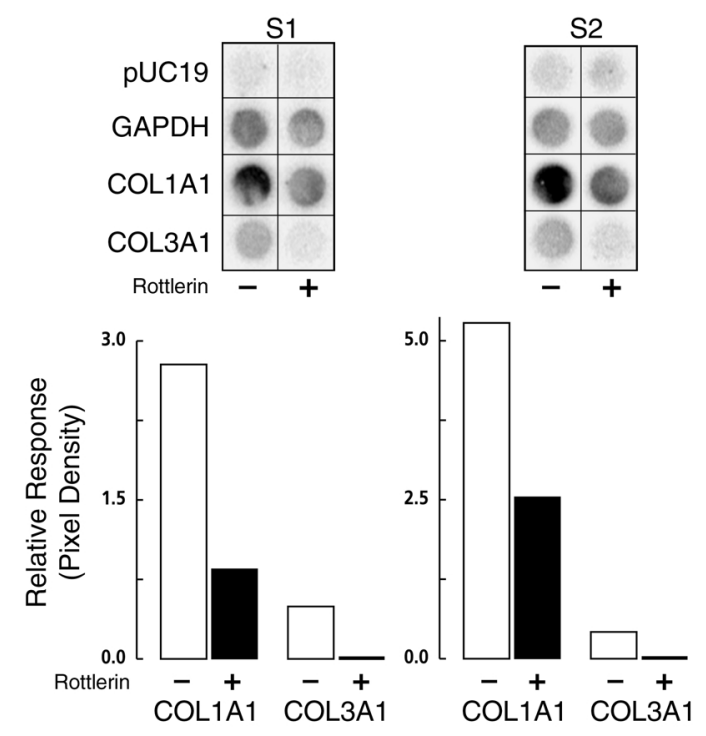

Figure 6

In vitro nuclear transcription of control or rottlerin-treated SSc cells. Confluent cultures of SSc fibroblasts (S1, S2) were incubated in T-175 flasks in MEM supplemented with 10\% (vol/vol) FBS and ascorbic acid $(50 \mu \mathrm{g} / \mathrm{ml})$ with or without rottlerin $(3 \mu \mathrm{M})$ for 24 hours. Nuclei were prepared and in vitro transcription assays were performed as described in Methods. Aliquots of ${ }^{32} \mathrm{P}$-labeled RNA containing equal cpm were hybridized to duplicate dot-blotted cDNAs for $\alpha 1$ (I) and $\alpha 1$ (III) procollagens, GAPDH, or the plasmid pUC19. After hybridization these were analyzed using storage phosphor technology. The values obtained were corrected for the transcription of GAPDH after subtraction of the background represented by PUC19. The values shown represent the corrected pixel densities from the average of two separate experiments. 
a Acetylated
Chloramphenicol
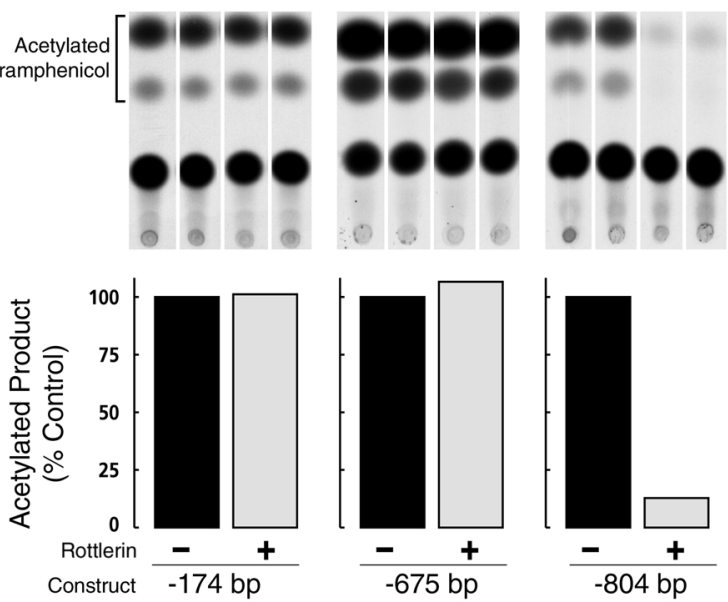

b
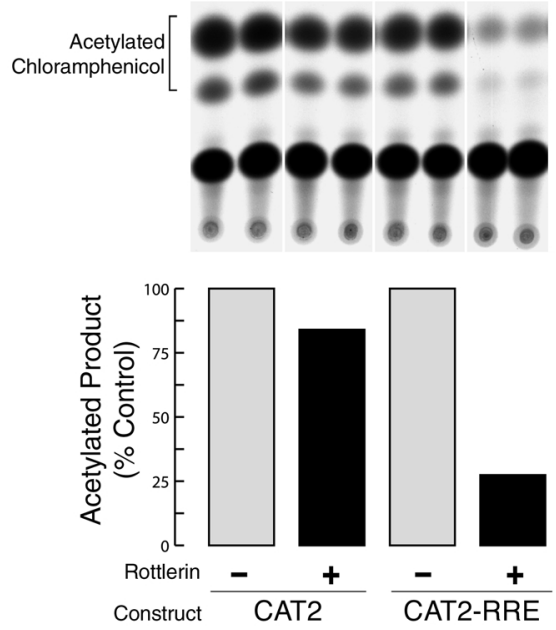

Figure 7

Identification of the COL1A1 promoter region responsive to rottlerin. (a) Autoradiogram of CAT assays performed with extracts from SSc cells that had been transfected with $2.5 \mu \mathrm{g}$ of one of various deletion constructs of COL1A 1 with $5^{\prime}$ endpoints at $-804,-675$, and -174 , and then incubated with $3 \mu \mathrm{M}$ rottlerin for 24 hours. The bar graph shows the densitometric scanning of the autoradiograms; the bars indicate arbitrary densitometric units of acetylated chloramphenicol. (b) Autoradiogram of CAT assays performed with extracts from SSc cells that had been transfected with $2.0 \mu \mathrm{g}$ of SJRott804 containing nucleotides -675 to -804 of the COL1A 1 promoter, cloned upstream of the heterologous herpes simplex virus TK promoter and treated with or without $3 \mu \mathrm{M}$ rottlerin for 24 hours after transfection. The bar graph shows the densitometric analysis of the autoradiograph showing the proportion of acetylated chloramphenicol. CAT2, PBL CAT2; CAT2-RRE, pBLL CAT2 with rottlerin response element.

We have recently undertaken studies to identify nonSmad pathways that may participate in the regulation of expression of genes that encode various extracellular matrix proteins by members of the TGF- $\beta$ family. We identified the following enzymes as possible components in the overall regulation of fibronectin, type I and III collagens, and elastin by TGF- $\beta 1$ : phosphatidylcholine-specific phospholipase C, PKC- $\delta$, and geranylgeranyl transferase I (16-18). While these reactions are best known for their potential roles in mediating cytokine and growth factor signal transduction, we postulate that they may also be involved in regulating the levels of constitutive expression of matrix genes, including type I and type III collagens, and that altered activity of one or more of these components may play an important role in the pathogenesis of SSc and other fibroproliferative diseases. In order to test this hypothesis, we are investigating the effect of modulating the intracellular activity of each of these enzymes on the constitutive expression of matrix protein genes in normal and SSc cells. In a recent study, we demonstrated that inhibition of prenylation catalyzed by geranylgeranyl transferase I resulted in profound and selective inhibition of the expression of types I and III collagen genes (19). We further demonstrated that these inhibitory effects were largely due to a reduction in the transcription rate of the type I procollagen gene (19).

The PKC family of proteins is comprised of at least ten isozymes with diverse functions that are involved in numerous important cellular processes. These proteins have been divided into three groups based on their structure, cofactor requirements, and cellular distribution (reviewed in refs. 37-39). Numerous studies have established that PKC activation is necessary for stimulation of gene expression in response to proinflammatory cytokines, such as IL-1 and TNFs (40, 41), and that these effects appear to be mediated by specific activa-
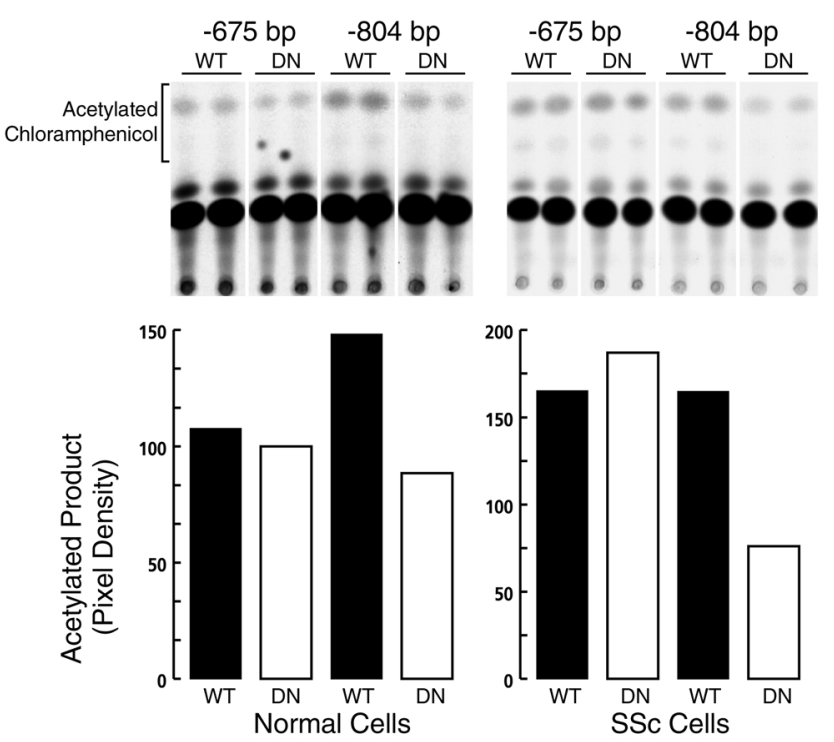

Figure 8

Cotransfection of COL1A1 promoter constructs and PKC- $\delta$ expression constructs into normal and SSc fibroblasts. Normal and SSc fibroblasts were cultured to $70 \%$ confluence and then cotransfected with 0.4 $\mu \mathrm{g}$ of either bp -675 or bp -804 COL1A1 deletion constructs and 0.4 $\mu \mathrm{g}$ of either a wild-type or a dominant-negative PKC- $\delta$ expression construct, as described in Methods. Following transfections, cell extracts were assayed for CAT activity. The upper panels show the autoradiograms of the CAT assays. The lower panels show the quantitation of CAT activity following normalization for the activity of the cotransfected $\beta$-galactosidase gene. Note that expression of the dominantnegative PKC- $\delta$ construct decreases the transcriptional activity of the bp -804 COL1A1 construct, but has no effect on that driven by the bp -675 COL1A1 construct. WT, wild-type; DN, dominant negative. 

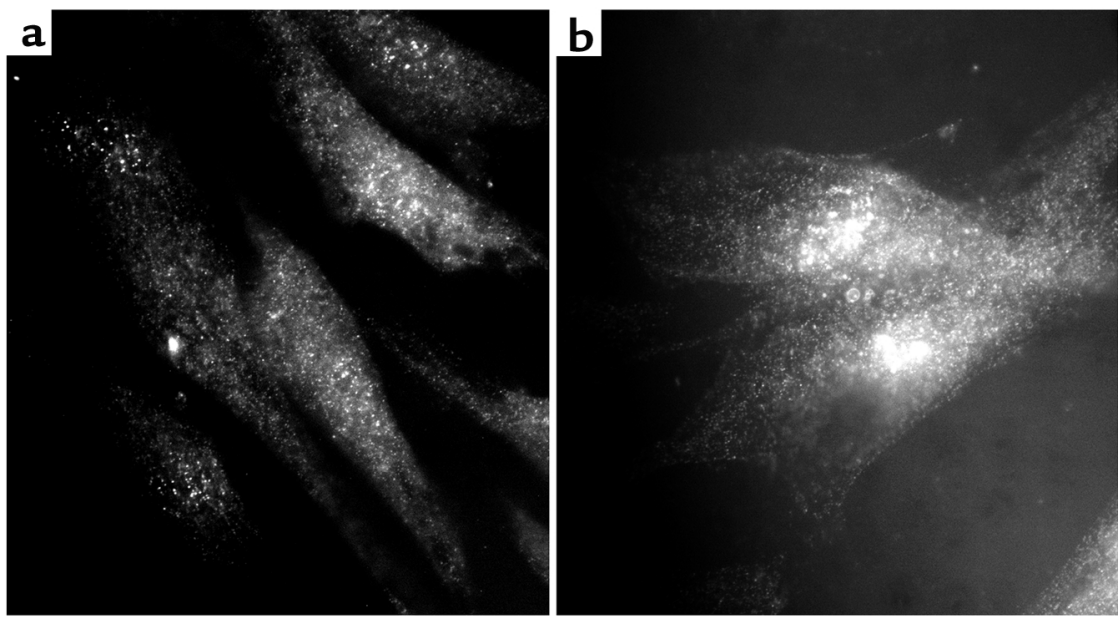

\section{Figure 9}

High-resolution fluorescence immunomicroscopy of normal and SSc fibroblasts with an anti-PKC- $\delta$ antibody. Normal and SSc fibroblasts were cultured on coverslips, and when confluent were processed for high-resolution fluorescence immunomicroscopy using a polyclonal anti-PKC- $\delta$ antibody as described in Methods. (a) Normal fibroblasts. (b) SSc fibroblasts. Note the higher immunofluorescence displayed by SSc fibroblasts in comparison with normal cells. tion of a variety of transcription factors, including AP-1 (42), ATF-2 (43), and NF-אB (44, 45). However, a role for these enzymes in the regulation of collagen gene expression has not been described previously, nor have their effects on transcription factors involved in stimulating the transcriptional activity of collagen genes such as Sp1 or CCAAT binding factor.

Here, we examined the possibility that PKC- $\delta$ may be involved in the upregulation of collagen gene expression in SSc by assessing the levels of PKC- $\delta$ in normal and $\mathrm{SSc}$ cells using high-resolution fluorescence immunomicroscopy and Western analyses of cell lysates. We also analyzed the effects of expression of a dominantnegative PKC- $\delta$ construct on the promoter activity of COL1A1. In addition, we examined the effects of the selective inhibition of this PKC isozyme on collagen gene expression in these cells. For the latter purpose, we used rottlerin, a compound shown previously to cause a potent inhibition of PKC- $\delta$ at concentrations that do not affect other PKCs $(20,21)$. The $\mathrm{IC}_{50}$ for $\mathrm{PKC}-\delta$ is $3-6$ $\mu \mathrm{M}$, whereas all other PKCs are not inhibited until concentrations become at least one order of magnitude higher (20). The results demonstrated that SSc cells contained higher levels of immunoreactive PKC- $\delta$ than do normal cells. Furthermore, expression of a dominant-negative $\mathrm{PKC}-\delta$ construct resulted in a substantial reduction in the promoter activity of COL1A1. The results using rottlerin showed that at low concentrations $(3 \mu \mathrm{M})$, it caused a potent inhibition of COL1A1 and COL1A3 expression in both cell types. In contrast, expression of the constitutively expressed cyclooxygenase- 1 and GAPDH genes was not affected, indicating that the inhibition was not due to a generalized toxic effect. In addition, there was no obvious cytotoxicity, as determined by trypan blue exclusion.

Our previous finding that inhibition of geranylgeranyl transferase I markedly reduced expression of types I and III collagens by normal and SSc fibroblasts suggested that a small prenylated GTPase belonging to the Ras superfamily may be involved in the regulation of collagen gene expression (19). Our current finding that inhibition of PKC- $\delta$ also resulted in a profound decrease in expression of type I and type III collagen raises the intriguing possibility that there may be a direct relationship between the activity of a small GTPase and PKC activation. Indeed, such a relationship has been proposed by Kamada et al. in a yeast system (46). Also, Hippensteil et al. have shown that inactivation of Rho, but not Ras proteins, prevented the translocation and activation of PKC- $\alpha$ in human endothelial and bronchial epithelial cells (47). These results support the notion that inhibition of geranylgeranyl transferase I might block the activation of PKC- $\delta$.

In the present study, we further demonstrated that rottlerin acts ultimately at the transcriptional level, and that its effect on COL1A1 expression is mediated through a 129-bp gene promoter segment encompassing nucleotides -675 to -804 . The critical role of this region was supported by the finding that expression of dominant-negative $\mathrm{PKC}-\delta$ inhibited the promoter activity of the bp -804 construct, but not that driven by the bp -675 construct. This region of the COL1A1 promoter does not contain regulatory elements that were previously considered to be involved in the transcriptional regulation of the gene. Thus, it is very likely that the rottlerin response is mediated by COL1A1 promoter elements not previously recognized as participants in the upregulation of collagen gene expression in SSc cells. Identification of these elements, which is currently in progress, could shed light on critical mech-

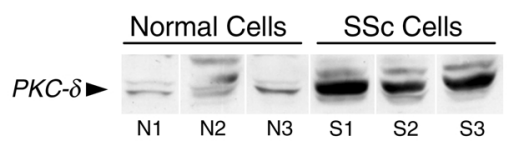

\section{Figure 10}

Western analysis of cell lysates from normal and SSc fibroblasts with an anti-PKC- $\delta$ antibody. Three cell lines of either normal or SSc dermal fibroblasts were cultured until confluent, and whole cell lysates were prepared as described in Methods. Aliquots of the lysates from each cell line were submitted to Western analysis using a polyclonal anti-PKC- $\delta$ antibody. Note that the main immunoreactive protein band (identified with the arrowhead) corresponding to PKC- $\delta$ is substantially increased in SSc fibroblasts compared with normal cells. 
anisms regulating interstitial collagen gene expression, both under normal conditions and in the course of fibroproliferative diseases. Our results also suggest that PKC- $\delta$ may be involved in the pathogenesis of the dysregulation of collagen gene expression that is characteristic of SSc. Further clarification of the putative role of PKC- $\delta$ in this process could provide valuable clues for the development of novel strategies for the treatment of deleterious fibrotic reactions, including those occurring in SSc.

\section{Acknowledgments}

We thank Weiqun Li for generously providing the PKC$\delta$ constructs. The expert assistance of Kate Salmon and Maria Mokan in the preparation of the manuscript is gratefully acknowledged. This work was supported by NIH grant AM-19616.

1. Medsger, T.A., Jr. 1997. Systemic sclerosis (scleroderma): clinical aspects. In: Arthritis and allied conditions. D.J. McCarty and W.J. Koopman, editors. Williams and Wilkins. Baltimore, Maryland, USA. 1433-1463.

2. Wigley, F.M. 1998. Systemic sclerosis. In: Rheumatology. H. Klippel and P.A. Dieppe, editors. Mosby International. London, United Kingdom. 9.1-9.14. 3. Batuman, O.A., and Jimenez, S.A. 1993. The molecular pathology of autoimmune diseases. Harwood Academic Publishers. Chur, Switzerland. 377 pp.

4. Jimenez, S.A., Hitraya, E., and Varga, J. 1996. Pathogenesis of scleroderma. Collagen. Rheum. Dis. Clin. North Am. 22:647-674.

5. D'Angelo, W.A., Fries, J.F., Masi, A.T., and Shulman, L.E. 1969. Pathologic observations in systemic sclerosis (scleroderma). A study of fifty-eight autopsy cases and fifty-eight matched controls. Am. J. Med. 46:428-440.

6. Hesselstrand, R., Scheja, A., and Akesson, A. 1998. Mortality and causes of death in a Swedish series of systemic sclerosis patients. Ann. Rheum. Dis. 57:682-686.

7. LeRoy, E.C. 1974. Increased collagen synthesis by scleroderma skin fibroblasts in vitro: a possible defect in the regulation or activation of the scleroderma fibroblast. J. Clin. Invest. 54:880-889.

8. Jimenez, S.A., Feldman, G., Bashey, R.I., Bienkowski, R., and Rosenbloom, J. 1986. Co-ordinate increase in the expression of type I and type III collagen genes in progressive systemic sclerosis. Biochem. J. 237:837-843.

9. Kikuchi, K., Smith, E.A., LeRoy, E.C., and Trojanowska, M. 1992. Direct demonstration of transcriptional activation of collagen gene expression in systemic sclerosis fibroblasts. Biochem. Biophys. Res. Commun. 187:45-50.

10. Hitraya, E.G., and Jimenez, S.A. 1996. Transcriptional activation of the $\alpha(\mathrm{I})$ procollagen gene in systemic sclerosis dermal fibroblasts. Role of intronic sequences. Arthritis Rheum. 39:1347-1354.

11. Border, W.A., and Noble, N.A. 1994. Transforming growth factor $\beta$ in tissue fibrosis. N. Engl. J. Med. 331:1286-1292.

12. Branton, M.H., and Kopp, J.B. 1999. TGF- $\beta$ and fibrosis. Microbes Infect. 1:1349-1365.

13. Smith, E.A., and LeRoy, E.C. 1990. A possible role for transforming growth factor- $\beta$ in systemic sclerosis. J. Invest. Dermatol. 95(Suppl.):125S-127S.

14. Massague, J., and Wotton, D. 2000. Transcriptional control by the TGF$\beta /$ Smad signaling system. EMBOJ. 19:1745-1754.

15. Attisano, L., and Wrana, J.L. 2000. Smads as transcriptional co-modulators. Curr. Opin. Cell Biol. 12:235-243.

16. Kucich, U., Rosenbloom, J., Abrams, W.R., Bashir, M.M., and Rosenbloom, J.C. 1997. Stabilization of elastin mRNA by TGF- $\beta$ : initial characterization of signaling pathway. Am.J. Respir. Cell. Mol. Biol. 17:10-16.

17. Kucich, U., et al. 1998. Requirement for geranylgeranyl transferase I and acyl transferase in the TGF- $\beta$-stimulated pathway leading to elastin mRNA stabilization. Biochem. Biophys. Res. Comm. 252:111-116.

18. Kucich, U., et al. 2000 . TGF- $\beta 1$ stimulation of fibronectin transcription in cultured human lung fibroblasts requires active geranylgeranyl transferase I, phosphatidylcholine-specific phospholipase C, protein kinase $C-\delta$, and p38, but not erk1/erk2. Arch. Biochem. Biophys. 374:313-324.

19. Rosenbloom, J., et al. 2000. Inhibition of type I collagen gene expression in normal and systemic sclerosis fibroblasts by a specific inhibitor of geranylgeranyl transferase I. Arthritis Rheum. 43:1624-1632.

20. Gschwendt, M., et al. 1994. Rottlerin, a novel protein kinase inhibitor. Biochem. Biophys. Res. Commun. 199:93-98.

21. Kontny, E., Kurowska, M., Szczepanska, K., and Maslinski, W. 2000. Rottlerin, a PKC isozyme-selective inhibitor, affects signaling events and cytokine production in human monocytes. J. Lenkoc. Biol. 67:249-258.

22. 1980. Preliminary criteria for the classification of systemic sclerosis (scleroderma). Subcommittee for scleroderma criteria of the American
Rheumatism Association Diagnostic and Therapeutic Criteria Committee. Arthritis Rheum. 23:581-590.

23. Bashey, R.I., and Jimenez, S.A. 1977. Increased sensitivity of scleroderma fibroblasts in culture to stimulation of protein and collagen sythesis by serum. Biochem. Biophys. Res. Commun. 76:1214-1222.

24. Jimenez, S.A, Freundlich, B., and Rosenbloom, J. 1984. Selective inhibition of human diploid fibroblast collagen synthesis by interferons. J. Clin. Invest. 74:1112-1116.

25. Diaz, A., Muñoz, E., Johnston, R., Korn, J.H., and Jimenez, S.A. 1993. Regulation of human lung fibroblast $\alpha 1(\mathrm{I})$ procollagen gene expression by tumor necrosis factor $\alpha$, interleukin-1 $\beta$, and prostaglandin E2. J. Biol. Chem. 268:10364-10371.

26. Bellon, G. 1985. Quantification and specific detection of collagenous proteins using an enzyme-linked immunosorbent assay and an immunoblotting for cyanogen bromide peptides. Anal. Biochem. 150:188-202.

27. Chomczynski, P., and Sacchi, S. 1987. Single-step method of RNA isolation by acid guanidinium thiocyanate-phenol-chloroform extraction. Anal. Biochem. 162:156-159.

28. Diaz, A., and Jimenez, S.A. 1997. Interferon- $\gamma$ regulates collagen and fibronectin gene expression by transcriptional and post-transcriptional mechanisms. Int. J. Biochem. Cell Biol. 29:251-260.

29. Saitta, B., Gaidarova, S., Cicchillitti, L., and Jimenez, S.A. 2000. CCAAT binding transcription factor binds and regulates human COL1A1 promoter activity in human dermal fibroblasts. Arthritis Rheum. 43:2219-2229.

30. Jimenez, S.A., et al. 1994. Functional analysis of human $\alpha 1(\mathrm{I})$ procollagen gene promoter. Differential activity in collagen-producing and non-producing cells and response to transforming growth factor- $\beta 1$. J. Biol. Chem. 269:12684-12691.

31. Artlett, C.M., Chen, S.-J., Varga, J., and Jimenez, S.A. 1998. Modulation of basal expression of the human $\alpha 1$ (I) procollagen gene (COL1A1) by tandem NF-1/Sp1 promoter elements in normal human dermal fibroblasts. Matrix Biol. 17:425-434.

32. Li, W., et al. 1996. Expression of an ATP binding mutant of PKC-delta inhibits Sis-induced transformation of NIH3T3 cells. Oncogene. 13:731-737.

33. Bradford, M.M. 1976. A rapid and sensitive method for the quantitation of microgram quantities of protein utilizing the principle of protein DNA binding. Anal. Biochem. 72:248-254.

34. Gorman, C.M., Moffet, O.S., and Howard, B.H. 1982. Recombinant genomes which express chloramphenicol acetyl tranferase in mammalian cells. Mol. Cell. Biol. 2:1044-1051.

35. Muñoz, E., Suri, D., Amini, S., Khalili, K., and Jimenez, S.A. 1995. Stimulation of $\alpha 1$ (I) procollagen gene expression in NIH-3T3 cells by the human $\mathrm{T}$ cell leukemia virus type 1 (HTLV-1) Tax gene. J. Clin. Invest. 96:2413-2420.

36. Derynck, R., and Feng, X.H. 1997. TGF- $\beta$ receptor signaling. Biochim. Biophys. Acta. 1332:F105-F150.

37. Buchner, K. 2000. The role of protein kinase $\mathrm{C}$ in the regulation of cell growth and in signaling to the cell nucleus. J. Cancer Res. Clin. Oncol. 126:1-11.

38. Mellor, H., and Parker, P.J. 1998. The extended protein kinase C superfamily. Biochem. J. 332:281-292.

39. Dekker, L.V., Palmer, R.H., and Parker, P.J. 1995. The protein kinase C and protein kinase C related gene families. Curr. Opin. Struct. Biol. 5:396-402.

40. Lin, C.H., et al. 2000. Involvement of protein kinase C- $\gamma$ in IL-1 $\beta$-induced cyclooxygenase- 2 expression in human pulmonary epithelial cells. Mol. Pharmacol. 57:36-43.

41. Chen, C.C., Sun, Y.T., Chen, J.J., and Chiu, K.T. 2000. TNF- $\alpha$-induced cyclooxygenase- 2 expression in human lung epithelial cells: involvement of the phospholipase C- $\gamma 2$, protein kinase C- $\alpha$, tyrosine kinase, NF- $\kappa B-$

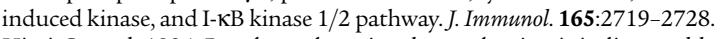

42. Hirai, S., et al. 1994. Ras-dependent signal transduction is indispensable but not sufficient for the activation of AP1/Jun by PKC $\delta$. EMBO J. 13:2331-2340.

43. Kawasaki, H., et al. 1998. p300 and ATF-2 are components of the DRF complex, which regulates retinoic acid- and E1A-mediated transcription of the c-jun gene in F9 cells. Genes Dev. 12:233-245.

44. Chen, C.C., Chen, J.J., and Chou, C.Y. 2000. Protein kinase C $\alpha$ but not $\mathrm{p} 44 / 42$ mitogen-activated protein kinase, $\mathrm{p} 38$, or c-Jun NH(2)-terminal kinase is required for intercellular adhesion molecule- 1 expression mediated by interleukin-1 $\beta$ : involvement of sequential activation of tyrosine

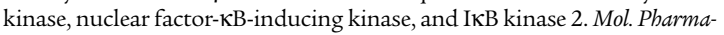
col. 58:1479-1489.

45. Chen, C.C., Wang, J.K., and Lin, S.B. 1998. Antisense oligonucleotides targeting protein kinase $C-\alpha,-\beta I$, or $-\delta$ but not $-\lambda$ inhibit lipopolysaccharideinduced nitric oxide synthase expression in RAW 264.7 macrophages: involvement of a nuclear factor $\mathrm{kB}$-dependent mechanism. J. Immunol. 161:6206-6214.

46. Kamada, Y., et al. 1996. Activation of yeast protein kinase C by Rho 1 GTPase. J. Biol. Chem. 271:9193-9196.

47. Hippensteil, S., et al. 1998. Rho protein inhibition blocks protein kinase C translocation and activation. Biochem. Biophys. Res. Comm. 245:830-834. 